\title{
Methods for selecting the supporting curve for the model of hysteresis loop
}

\author{
O.N. Korsun ${ }^{1}$ and A.V. Stulovskii ${ }^{1, *}$ \\ ${ }^{1}$ State Research Institute of Aviation Systems, Moscow, Russia
}

\begin{abstract}
The article deals with a model describing the dependence of aerodynamic coefficients on the angle of attack for post-stall conditions. This paper also discusses the choice of parameters for the calculating the lift coefficient in such cases. In addition, it also considers some methods used to choose the shape of a supporting curve. The article also provides arguments concerning the physical interpretation of the coordinate of flow separation point in the implementation of the model. The examples of processing the flight test data are presented.
\end{abstract}

\section{Introduction}

It is well known, that the aerodynamic processes at overcritical values of the angle of attack have fundamental differences if compared with similar processes occurring in the operational range of attack angles [1].

One of the most important features of this mode is the presence of hysteresis in the dependence of the aerodynamic coefficients on the angle of attack. Asymmetry of the flow disruption and its recovery during variation of the angle of attack explains this phenomenon. Since it takes place even with a very small value of the derivative of the angle of attack, such fact was named static hysteresis.

\section{Mathematical model of hysteresis for the lift coefficient}

Of considerable interest is the construction of models describing the behavior of the lift coefficient for the overcritical values of the angle of attack. The estimation of the coefficient itself was obtained through the identification methods described in [2], using the model [3] of forces and torques generated by thrust vector deviation.

One type of such model is given in [1, 4]. More detailed discussion concerning this topic may be found in [5-7]. This model includes additional parameter $\bar{x}-$ the coordinate characterizing the position of the flow separation point on the wing profile. Its units of measurement are the chord shares. Therefore, its value ranges from 0 to 1 . The model itself is described by a system of two equations:

$$
\begin{aligned}
& c_{y e}(\alpha)=c_{y 0}(\alpha)\left(\frac{1+\sqrt{\bar{x}}}{2}\right)^{2} ; \\
& \tau_{1} \frac{d \bar{x}}{d t}+\bar{x}=x_{0}(\alpha), \quad \bar{x}(0)=1,
\end{aligned}
$$

where $c_{y 0}(\alpha)$ - estimation of the lift coefficient values as the function of the angle of attack, with no account for hysteresis; $\tau_{1}=$ const - model parameter; $x_{0}(\alpha)-$ static dependence of the co-ordinate of the flow separation point from the angle of attack.

The introduction of flow separation point makes possible the description of the asymmetry of the recovery and the disruption of the flow.

By the form of the expression itself it becomes clear that the model needs to be adapted for practical use - the function $x_{0}(\alpha)$ should be chosen. One of the possible function, according to [7], is

$$
x_{0}(\alpha)=0.5\left\{1-\tanh \left[\lambda\left(\alpha-\tau_{2} d \alpha / d t-\alpha^{*}\right)\right]\right\},
$$

where $d \alpha / d t$ - time derivative of the angle of attack, $\mathrm{rad} / \mathrm{s} ; \tau_{1}, \tau_{2}, \alpha^{*}, \lambda$ - parameters of the equation, which values are determined by the characteristics of the aerodynamic profile and the configuration of the wing, as well as by the dynamics of the flow process itself.

The physical sense of these parameters is discussed in more detail in [7]. Nonetheless, the question concerning choice of $c_{y 0}(\alpha)$ remains actual, and the next section answers it.

\section{Supporting curve}

Physical sense of the $c_{y 0}(\alpha)$ becomes clear after more detailed examination of the system (1). It could be seen that $c_{y 0}(\alpha)$ function serves as a basic, or supporting, curve and values of $c_{y e}(\alpha)$ are derived from it by

Corresponding author: avstlv2@gmail.com 
addition of disturbances depending on coordinate of the flow separation point $\bar{x}$.

Cubic Hermite splines were used for specifying this curve, since they are capable of producing a sufficiently wide range of curves. Here we briefly give the formulas used for their construction. The reader can find more about them, as well as about the properties of these splines, for example, in [8].

Let us assume that for the certain interval $\left[x_{1}, x_{M}\right]$ $M$ values of the independent variable are given, that is $x_{j}, j=\overline{1, M}$. Each such point is called spline node. Then the value of the spline for an arbitrary point $x \in\left[x_{i}, x_{i+1}\right]$ is obtained by the formula

$$
S(x)=\varphi_{1}(t) f_{i}+\varphi_{2}(t) f_{i+1}+\varphi_{3}(t) h_{i} f_{i}^{\prime}+\varphi_{4}(t) h_{i} f_{i+1}^{\prime},
$$

where

$$
\begin{aligned}
& \varphi_{1}(t)=(1-t)^{2}(1+2 t), \\
& \varphi_{2}(t)=t^{2}(3-2 t), \\
& \varphi_{3}(t)=t(1-t)^{2}, \\
& \varphi_{4}(t)=-t^{2}(1-t), \\
& h_{i}=x_{i+1}-x_{i}, \\
& t=\left(x-x_{i}\right) / h_{i} .
\end{aligned}
$$

Now let us turn back to the model of aerodynamic hysteresis. Considering the system (1) mathematically it can be concluded that values of $c_{y e}(\alpha)$ will invariably be lower then $c_{y 0}(\alpha)$ values.

This fact was additionally confirmed by building the supporting curve passing through the center of the hysteresis loop (Figure 1). Then the parameter identification of hysteresis model (1) was carried out. The results presented in Figure 1 show that the fits the lower branch of the loop with high precision, while the upper branch remains unreachable.

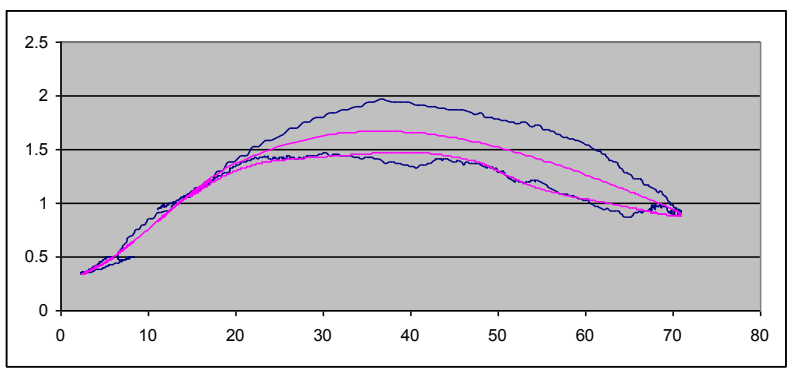

Fig. 1. The values of the lift coefficient (blue line) and the values obtained using the hysteresis model with the supporting curve passing in the middle of the loop (violet line), depending on the angles of attack.

Based on this observation, decision was made to use the upper branch of the hysteresis loop as the supporting curve. The resulting approximation proved to be excellent as it is shown in Figure 2.

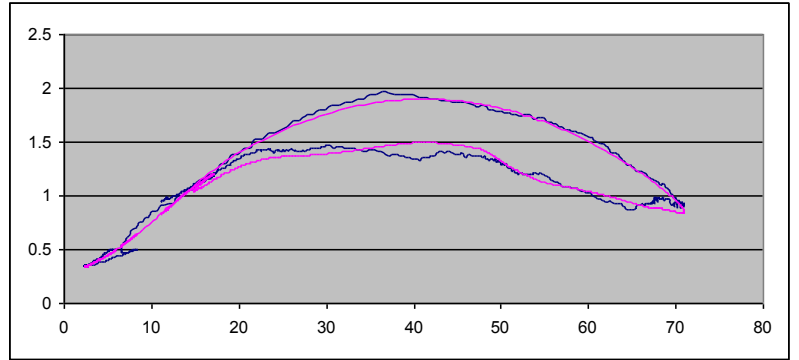

Fig. 2. The values of the lift coefficient (blue line) and the values obtained using the hysteresis model with the supporting curve passing on the upper branch of the loop (violet line), depending on the angles of attack.

\section{Procedure of curve selection}

As mentioned above, $c_{y 0}(\alpha)$ is interpreted as the value of the lift coefficient for steady flow. But it is known in aerodynamics that a partial flow separation starts just when angles of attack exceed overcritical value.

As could be seen in Figure 2, choice of upper branch of hysteresis loop produces sufficiently good description of the experimental data. However, variation of the coordinate of the flow separation point in this case shows that separation of the flow starts at angles of attack far beyond critical value (Figure 3, blue line). This result is contrary to the nature of the process.

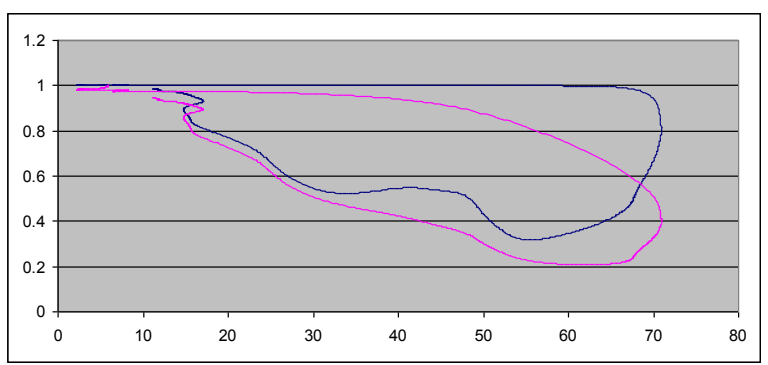

Fig. 3. The values of the flow separation coordinates depending on angle of attack for supporting curve from figure 2 (blue line) and figure 4 (violet line).

Therefore, we can conclude that the supporting curve should pass higher than the upper branch of the loop. The bifurcation point should correspond to the angle of attack critical value. For example, let us consider the supporting curve passing as shown in Figure 4.

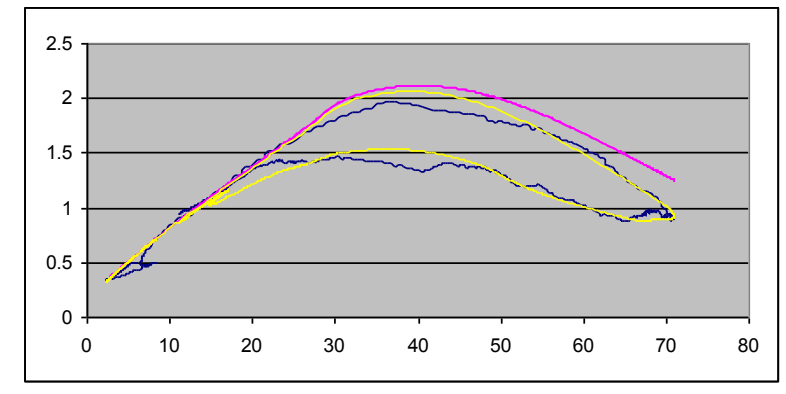

Fig. 4. The values of the lift coefficient (blue line) and the values obtained using the hysteresis model (yellow line) with the supporting curve (violet line) passing above the hysteresis loop, depending on the angles of attack. 
In this case the variation of the flow separation point coordinate looks more natural, as shown in Figure 3, violet line. At the same time the loop approximation is still very accurate. Therefore, this kind of supporting curve provides good opportunities for tuning the hysteresis model parameters. It should be also noted that the supporting curve constructed above the upper branch of hysteresis loop is in certain sense virtual since it does not correspond to any actual aerodynamic dependency.

So, it is evident that considerable room for choosing a particular supporting curve exists.

\section{Conclusions}

The paper shows that there are two principal modes to choose the supporting curve in the aerodynamic hysteresis model.

The simplest way is to draw the curve following the upper branch of the hysteresis loop. In this case we obtain a good approximation of the loop, but the variation of the flow separation point coordinate contradicts the physical nature of the process. It may be acceptable when the flow separation point is not under consideration.

Another way is to construct the virtual supporting curve positioned above the upper branch of the loop. In this case both the loop approximation and the separation point variations are acceptable. The problem is that due to the considerable room for constructing the curve the choice is arbitrary to certain extent.
This work is supported by Russian Foundation for Basic Research (RFBR), project 17-08-00856-a.

\section{References}

1. G.S. Bjushgensa (eds) Ajerodinamika, ustojchivost' $i$ upravljaemost' sverhzvukovyh samoletov, (Moscow: Nauka, 1998)

2. A.V. Kanyshev, O.N. Korsun, A.V. Stulovskii, Mehatronika, avtomatizacija, upravlenie, 19, 201 (2018)

3. A.V. Kanyshev, O.N. Korsun, A.V. Stulovskii, ITM Web of Conference, 10, 01004 (2017)

4. M.G. Goman, A.N. Khrabrov, Journal of Aircraft, 31, 1109 (1994)

5. D.M. Luchtenburg, C.M. Rowley, M.W. Lohry, L. Martinelli, R.F. Stengel, Journal of Aircraft, 52, 890 (2015)

6. R.V. Jategaonkar, Flight vehicle system identification: A time domain methodology, (Reston: AIAA, 2006)

7. V.N. Ovcharenko, Identifikacija ajerodinamicheskih harakteristik vozdushnyh sudov po poletnym dannym (Moscow: Izdatel'stvo MAI, 2017)

8. Ju.S. Zav'jalov, B.I. Kvasov, V.L. Miroshnichenko, Metody splain-funkcyj (Moscow: Nauka, 1980) 